\title{
Endogenous Timing and the Choice of Quality in a Vertically Di Rerentiated
}

\author{
Duopoly \\ Luca Lambertini \\ Department of Economics \\ University of B ologna \\ Strada Maggiore 45 \\ I-40125 Bologna, Italy \\ fax 39-51-6402664 \\ e-mail lamberti@spbo.unibo.it
}

September 28, 1998

A cknowledgements. This paper was written when I was at the Institute of Economics, 


\begin{abstract}
A bstract
The endogenous choice of timing is discussed in a vertically di ßerentiated duopoly where quality improvement requires a ${ }^{-}$xed convex cost. The timing decision concerns the quality stage. Using an extended game with observable delay, it is shown that only simultaneous equilibria can arise. This puts into question the ability of Stackelberg games to describe the entry process.

J .E.L. classi ${ }^{-}$cation: D21, D43, L13
\end{abstract}

K eywords: product quality, $R \& D$ investment, timing, extended game

\title{
1. Introduction
}

A n established result in the theory of oligopoly under vertical di ßerentiation states that the high-quality earns higher pro $^{-}$ts than low-quality rivals (see, inter alia, Gabszewicz and Thisse, 1979, 1980; Shaked and Sutton, 1982, 1983; Donnenfeld and Weber, 1995). A general proof of this result for every convex - xed-cost function of quality improvement is provided by Lehmann-Grube (1997). Aoki and Prusa (1997) adopt a speci ${ }^{-}$c case of the cost function analysed by LehmannGrube, to investigate the consequences on $\mathrm{pro}^{-} \mathrm{ts}$, consumer surplus and social

University of Copenhagen. I thank an associate editor, an anonymous referee and Dan Sasaki for helpful comments. The usual disclaimer applies. 
welfare of the timing of investment in product quality in a vertically di ßerentiated duopoly where the market stage is played in the price space. They show that (a) sequential play induces both " $r m s$ to invest less in product quality, as compared to what they would if they were to play simultaneously; (b) under sequential play, the leader prefers to supply the high-quality good; and (c) industry pro ${ }^{-}$ts are higher under sequential play than under simultaneous play, while the opposite obviously holds for social welfare. Hence, the authors suggest that ${ }^{-} \mathrm{rms}$ may enjoy coordination bene- ts under sequential quality choice. Though, since the - rm supplying the high-quality good prefers sequential play to simultaneous play, while the opposite applies to the ${ }^{-} \mathrm{rm}$ producing the low-quality good, ${ }^{-}$rms can coordinate over sequential play if either (i) a side payment is possible; or (ii) ${ }^{-} \mathrm{rms}$ discover and introduce new varieties at random. In the latter perspective, the authors identify a probability interval in which both ${ }^{-}$rms prefer the sequential move game.

Although suggestive and indeed acceptable, neither of the two routes appear fully satisfactory, for di ßerent reasons. The possibility of a side payment raises the issue of opportunistic behaviour on the part of both ${ }^{-} \mathrm{rms}$. As to the probabilistic approach, one would like to exploit it fully from the outset, and analyse the game as a stochastic $R \& D$ race. In the original paper, qualities are produced 
in a deterministic environment. M oreover, random success in $R \& D$ activity puts strongly into question point (b). If innovation is stochastic, there is no reason to believe a priori that the leader will supply the high-quality good. As an example, consider the car industry. An innovation in the form of a new family car in the range of station wagons may be easier to accomplish than a new ${ }^{\circ}$ agship sportcar, so that the leader may well end up with the low-quality good. This points to a possible tradeo ${ }^{\circledR}$ between early innovation and the attainment of a dominant position in the market (see Dutta, Lach and R ustichini, 1995). ${ }^{1}$

My purpose is to proceed to a selection between simultaneous and sequential quality choice by embedding quality competition into an extended game with observable delay (Hamilton and Slutsky, 1990; HS henceforth), where players (say, - rms) are required to set both the actual moves or actions in the quality space, and the time at which such actions are to be implemented. When ${ }^{-}$rms choose to act at di Rerent times, sequential equilibria obtain, while if they decide to move at the same time, simultaneous Nash equilibria are observed. For sequential play to emerge at the subgame perfect equilibrium of the extended game, its outcome must at least weakly Pareto-dominate the outcome associated with simultaneous

\footnotetext{
${ }^{1}$ For the analysis of the incentive for the low-quality ${ }^{-} r m$ towards leapfrogging, see R osenkranz (1997).
} 
moves. The choice of timing occurs in a preplay stage which does not take place in real time, so that there is no discounting associated with waiting and payo®S are the same whether ${ }^{-}$rms choose to move as soon as possible or they delay as long as they can. The decision to play early or at a later time is not su \pm cient per se to yield sequential play, since an analogous decision taken by the rival leads to simultaneous play. In the present setting, another extension can be envisaged, namely, the one concerning the choice between high and low quality, i.e., the relative positions of ${ }^{-} \mathrm{rms}$ in the product space. Therefore, I shall describe two alternative symmetric extended games, resulting from the permutation of the stages describing the timing choice and the location of ${ }^{-} \mathrm{rms}$ along the quality spectrum, respectively. It turns out that the subgame perfect equilibrium of both games entails simultaneous play at the quality stage, so that Stackelberg outcomes are ruled out. This result, which appears to be in contrast with ' ${ }^{-}$ms' behaviour in the real world, may cast a shadow on the reliability of this approach as a stylised description of the process of innovation and entry.

The remainder of the paper is structured as follows. The basic model of vertical di ßerentiation is summarized in section 2. Section 3 describes the extended games. Finally, section 4 provides concluding remarks. 


\section{The vertical di Rerentiation model}

Here I shall brie ${ }^{\circ}$ y resume the basic setting. T wo single-product ${ }^{-} \mathrm{rms}$, labelled as 1 and 2, produce goods of di ßerent qualities, $q_{1}$ and $q_{2} 2[0 ; 1)$, through the same technology, $C\left(q_{i}\right)=k q_{i}^{2}$; with $k>0$ : This can be interpreted as ${ }^{-}$xed cost due to the $R \& D$ e ${ }^{\circledR}$ rt needed to produce a certain quality, while variable production costs are assumed away. P roducts are o ßered on a market where consumers have unit demands, and buy if and only if the net surplus from consumption $v_{\mu}\left(q_{i} ; p_{i}\right)=$ $\mu q_{i} i p_{i}, 0$; where $p_{i}$ is the unit price of the good of quality $q_{i}$, purchased by a generic consumer whose marginal willingness to pay is $\mu 2[0 ; \bar{\mu}]: 2$ I assume that $\mu$ is uniformly distributed with density one over such interval, so that the total mass of consumer is $\bar{\mu}$. Firms compete in two stages, the ${ }^{-}$rst being played in the quality space, the second in the price space. I maintain A oki and Prusa's assumption that downstream Bertrand competition is simultaneous. Hence, proceeding by backward induction, the pro ${ }^{-} \mathrm{t}$ function of ${ }^{-} \mathrm{rm} 1$ at the ${ }^{-}$rst stage looks as follows (cf. A oki and Prusa, 1997: Lemma 1, p. 106):

$$
1 / 4\left(q_{1} ; q_{2}\right)=\frac{4 \bar{\mu}^{2} q_{1}^{2}\left(q_{1} ; q_{2}\right)}{\left(4 q_{1} ; q_{2}\right)^{2}} ; k q_{1}^{2} \text { if } q_{1}, q_{2} ;
$$

\footnotetext{
${ }^{2}$ A oki and Prusa (1997: p. 106) normalise the support to $[0,1]$.
} 


$$
1 / 4\left(q_{1} ; q_{2}\right)=\frac{\bar{\mu}^{2} q_{1} q_{2}\left(q_{2} i q_{1}\right)}{\left(4 q_{2} i q_{1}\right)^{2}} ; k q_{1}^{2} \text { if } q_{1}<q_{2}:
$$

A slight generalization of A oki and Prusa's results in terms of equilibrium qualities, pro ${ }^{-} t$ and social welfare can be given on the basis of (1) and (2). Depending on the entry sequence, ${ }^{-}$rms will alternatively take the high-quality $(\mathrm{H})$ or the low-quality (L) position. Table 1 summarises the outcomes associated with simultaneous play $(n)$, sequential play with the high-quality ${ }^{-} \mathrm{rm}$ leading $(\mathrm{HI})$, and sequential play with the low-quality ${ }^{-} \mathrm{rm}$ leading (LI), respectively. ${ }^{3}$

INSERT TABLE 1 HERE

Observe that

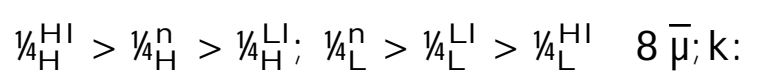

The above inequalities imply that ${ }^{-} \mathrm{rms}$ ' choice of the respective roles is independent of the ratio between the size of the market and the amount of ${ }^{-}$xed costs. M oreover, on the basis of social welfare levels in the three cases, it is worth em-

\footnotetext{
${ }^{3} \mathrm{~A}$ proof for the case where $\mathrm{k}=1=2$ is given by $\mathrm{M}$ otta (1993: pp. 116-117). Its generalisation is a matter of straightforward calculations. M or eover, the absolute level of $k$ is unimportant, in that the main results hold independently of the relative size of $k$ and $\bar{\mu}$ :
} 
phasizing that a public agency taking care of social surplus without distributive concerns should favour the leadership by the low-quality ${ }^{-} \mathrm{rm}$, while the leader always prefer to enter with a superior quality. This di ®erence in the structure of preferences can be traced back to the fact that, when leading, the low-quality - rm provides a higher quality than in any other situation.

\section{The extended game with observable delay}

Consider the following generalization of the extended game proposed by HS (1990:

p. 32), where ${ }^{-}$rms can set a single strategic variable in the basic game and must choose between moving ${ }^{-}$rst or second. ${ }^{4}$ Here, given simultaneous play in the price stage, the basic stage game is played in the quality space, and two extensions are accounted for. De ne as $i=(I ;-; \S ; T ; Q ; \mid)$ the extended game with observable delay, where all decisions are taken non-cooperatively. The set of players ( ${ }^{-}{ }^{-} \mathrm{rms}$ ) is I $=f 1 ; 2 g$, and $q_{1}$ and $q_{2} 2 Q=[0 ; 1)$ are the intervals of $R$ representing the actions available to players 1 and 2 in the basic game. Payo ${ }^{\circledR S}$ (pro ${ }^{-} t$ levels) ${ }^{1 / 4}$ and $1 / 42$ depend on the actions undertaken in the quality stage, so that $1 / 4$ and

\footnotetext{
${ }^{4} \mathrm{HS}$ (1990) also propose an extended game with action commitment, where each player must commit to a speci- $\mathrm{c}$ action irrespectively of the rival trying to lead or follow. The equilibrium of such a game is never unique; in particular, it always allows for sequential moves (see their Theorem VII, p. 42).
} 
$1 / \mathbb{q}: q_{1} f q_{2} ! R$. Accordingly, $\mid=f^{1 / 4} ; ; j k\left(q_{i}^{j}\right)^{2} g ;$ where $j=f n ;|f ; f| g$ denotes the outcome according to the timing combination. The set of times at which ${ }^{-}{ }^{-} \mathrm{ms}$ can choose to move is $\mathrm{T}=\mathrm{fF} ; \mathrm{Sg}$, i.e., ${ }^{-}$rst or second. The set of strategies for player i $2 \mathrm{I}$ is $\S_{i}=f F ; \operatorname{Sg} f \Theta_{i}$, where $\Theta_{i}$ is the function that maps $f T f q_{2}$ (or $\left.q_{1}\right)$ g into $q_{1}\left(\right.$ or $\left.q_{2}\right)$. If both ${ }^{-} r m s$ choose to move at the same time, they obtain the payo ${ }^{\circledR S}$ associated with the simultaneous $N$ ash equilibrium, otherwise they get the payo $\mathbb{}$ S associated with the Stackelberg equilibrium, e.g., if 1 moves ${ }^{-}$rst and 2 moves second, or vice versa. Finally, the presence of $i k\left(q_{i}^{j}\right)^{2}$ remains to explain. This leads us to the choice between o from which ${ }^{-} \mathrm{rms}$ can choose is - $=\mathrm{fH} ; \mathrm{Lg}$; where $\mathrm{H}$ and $\mathrm{L}$ stand for high and low quality, respectively. The set of strategies for player $i$ is $\underline{a}_{i}=f H ; \operatorname{Lg} f x_{i}$, where $x_{i}$ is the function that maps $f-f q_{2}\left(\right.$ or $\left.q_{1}\right) g$ into $q_{1}\left(\right.$ or $\left.q_{2}\right)$. When both choose $H$ (or $L$ ) they incur a loss corresponding to the $R \& D$ e $\mathbb{B}$ ort independently of timing, otherwise they obtain the pro ${ }^{-} t$ determined by their quality levels as well as timing.

I am now in a position to describe the two alternative extended games with observable delay that can be conceived through a permutation of the choice of timing and the choice of location along the quality spectrum. The ${ }^{-}$rst extended game arises if ${ }^{-} \mathrm{rms}$ set the sequence of moves before choosing between high and 
low quality. The second obtains when the order of such choices is reversed. The results of both games are summarized by the following ${ }^{5}$

Proposition 1. The subgame perfect equilibrium of an extended game with observable delay, where ${ }^{-}$rms have to determine the timing of moves in the quality stage as well as their distribution along the quality range, involves simultaneous play regardless of the sequence in which such decisions are taken.

Proof. The ${ }^{-}$rst extended game, where ${ }^{-}{ }^{-}$ms set the timing before proceeding to choose which quality to produce, is described by matrix 1. In each cell, the ${ }^{-}$rst payo ${ }^{\circledR}$ refers to ${ }^{-} r m$ 1, the second to ${ }^{-} r m$ 2. The cells where payo ${ }^{\circledR S}$ are $(i ; i)$ represent all cases where ${ }^{-}$rms enter with identical quality, so that revenues are nil by virtue of the Bertrand paradox and each ${ }^{-} r m$ looses $R \& D$ costs.

\section{INSERT MATRIX 1 HERE}

On the basis of the inequalities in (3), matrix 1 can be easily reduced to a $2 f$ 2 form, as follows. Consider the north-west quadrant, describing the subgame

\footnotetext{
5It can be shown that the same result holds if ${ }^{-} r m$ i's total cost were $C\left(q ; x_{i}\right)=k x_{i} q^{2} ; x_{i}$ being the output level (see Lambertini, 1996).
} 
where both ${ }^{-}$rms move as early as possible, and then choose which quality to supply. Both $(\mathrm{L} ; \mathrm{H})$ and $(\mathrm{H} ; \mathrm{L})$ are Nash equilibria of the subgame, therefore in the remainder I shall use the pro ${ }^{-}$ts resulting from the correlated equilibrium, i.e., $(1 / 4+1 /$ $1 / 2)=2=0: 064915 \bar{\mu}^{-4}=k: 6$ The remaining quadrants of matrix 1 can be

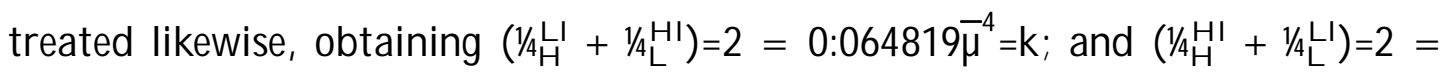
$0: 064993 \bar{\mu}^{4}=k:$ The reduced form is represented by matrix $1 b$.

\section{INSERT MATRIX Ib HERE}

It immediately appears that $\mathrm{F}$ is a strictly dominant strategy for both ${ }^{-} \mathrm{rms}$, so that the subgame perfect equilibrium of this extended game is identi ${ }^{-}$ed by the strategy pair $(F ; F)$, entailing that both ${ }^{-} r m s$ introduce their respective goods as early as possible, i.e., they play simultaneously in the quality stage. The equilibrium pro $^{-}$ts are those yielded by the correlated equilibrium of the fully simultaneous subgame, $\left(0: 064915 \bar{\mu}^{4}=k ; 0: 064915 \bar{\mu}^{4}=k\right)$.

Turn now to the extended game where ${ }^{-}$rms $^{-}{ }^{-}$sst decide over their location in

\footnotetext{
${ }^{6} \mathrm{~T}$ he existence of multiple equilibria yielded by the simple permutation of ${ }^{-} \mathrm{rms}$ reveals that there also exist mixed-strategy equilibria where ${ }^{-} r m s$ have a strictly positive probability to enter the market with the same quality level. Using the payoßs associated with correlated instead of mixed-strategy equilibria does not a eect the conclusions.
} 
the quality range, and then proceed to set the timing. This game is depicted in matrix 2.

\section{INSERT MATRIX 2 HERE}

Matrix 2 can be reduced by the same method applied in the previous case, by analysing each quadrant in isolation. This yields matrix $2 \mathrm{~b}$. Even without any inference concerning ' $r m s^{\prime}$ choices in the north-west and south-east quadrants of matrix 2 , it is worth observing that matrix $2 \mathrm{~b}$ looks as if ${ }^{-} \mathrm{rms}$ were al ways moving at the earliest occasion.

INSERT MATRIX 2b HERE

Matrix $2 b$ exhibits two Nash equilibria, namely $(H ; L)$ and $(L ; H)$, where, again, - $r$ ms move simultaneously. This obviously entails that as the representative payo ${ }^{\circledR}$ one may consider that associated with the correlated equilibrium identi ${ }^{-}$ed above, while discussing matrix 1 , i.e., $\left(0: 064915 \bar{\mu}^{4}=k ; 0: 064915 \bar{\mu}^{4}=k\right)$.

The result stated in Proposition 1 is clearly at odd with reality, where we 
usually observe ${ }^{-}$rms entering sequentially. This leads to reassess the reliability of a one-shot model as a tool to describe real world events. On the one hand, the approach adopted by Aoki and Prusa (1997) lacks a solid game-theoretical basis; on the other, the extended game approach adopted here excludes a result which we are well accustomed with in everyday's life. In either case, it appears that the essence of Stackelberg equilibria is their ability to capture the strategic use on the part of $a^{-} r m$ of the information contained in the rival's reaction function in the relevant space, while a more ${ }^{\circ}$ edged formalisation of both the innovation and the entry processes must take into account the role of real time and uncertainty in the R\&D activity, as in Dutta, Lach and Rustichini (1995).

From a social standpoint, the above analysis entails a clearcut conclusion, namely, that strategic interaction drives ${ }^{-}$rms towards an equilibrium which is collectively more desirable than the Stackelberg one where the high-quality ${ }^{-} \mathrm{rm}$ takes the lead. F inally, the highest level of social welfare, which would be generated by appointing the low-quality ${ }^{-} \mathrm{rm}$ as the leader, remains out of reach unless an intervention is designed to induce it. ${ }^{7}$

\footnotetext{
${ }^{7} \mathrm{Or}$ at least to mimic such a result through the introduction of a minimum quality standard (see Ronnen, 1991; Boom, 1995; Crampes and Hollander, 1995; Ecchia and Lambertini, 1997, inter alia).
} 


\section{Concluding remarks}

In this note, I have re-examined an issue tackled by A oki and Prusa (1997), under a new perspective, namely, embedding quality competition into an extended game with observable delay in the spirit of Hamilton and Slutsky (1990). When ${ }^{-} \mathrm{rms}$ are given the possibility of non-cooperatively setting the timing of moves as well as their location along the quality spectrum, and such choices can be taken in this or the opposite sequence, then sequential play is ruled out and ${ }^{-} \mathrm{rms}$ are driven by individual rationality to set qualities simultaneously. ${ }^{8}$ This leads to a drastic reconsideration of the descriptive power of the widely adopted one-shot two-stage model of vertical di ßerentiation, in that an approach to the description of the entry process based on either (i) a comparative evaluation of Stackelberg vs Nash equilibria on industry basis (as in A oki and Prusa, 1997), or (ii) a selection mechanism between them (as here), appears unable to describe in realistic terms the observed behaviour of ${ }^{-} \mathrm{rms}$.

\footnotetext{
8It remains true, however, that an extended game with action commitment would allow for sequential moves to characterize one or more equilibria of the game, alongside with the simultaneous equilibria derived here.
} 


\section{R eferences}

[1] A oki, R. \& Prusa, T. (1997). Sequential versus simultaneous choice with endogenous quality. International J ournal of Industrial Organization, 15, 103121.

[2] B oom, A. (1995). A symmetric international minimum quality standards and vertical di ßerentiation. J ournal of Industrial Economics, 43, 101-19.

[3] Crampes, C. \& Hollander, A. (1995). Duopoly and quality standards. European Economic Review, 39, 71-82.

[4] Donnenfeld, S. \& Weber, S. (1995). Limit qualities and entry deterrence. RAND J ournal of Economics, 26, 113-30.

[5] Dutta, P.K ., Lach, S. \& Rustichini, A. (1995). B etter late than early: vertical di ßerentiation in the adoption of a new technology. J ournal of E conomics and M anagement Strategy, 4, 563-589.

[6] Ecchia, G. \& Lambertini, L. (1997). Minimum quality standards and collusion. J ournal of Industrial E conomics, 45, 101-113. 
[7] Gabszewicz, J .J . \& Thisse, J .-F . (1979). Price competition, quality and income disparities. J ournal of Economic Theory, 20, 340-359.

[8] Gabszewicz, J .J . \& Thisse, J .-F . (1980). Entry (and exit) in a di ßerentiated industry. J ournal of E conomic Theory, 22, 327-38.

[9] Hamilton, J. \& Slutsky, S. (1990). Endogenous timing in duopoly games: Stackelberg or Cournot equilibria. Games and E conomic B ehavior, 2, 29-47.

[10] Lambertini, L. (1996). Choosing roles in a duopoly for endogenously di ßerentiated products. Australian E conomic Papers, 35, 205-24.

[11] Lehmann-G rube, U. (1997). Strategic choice of quality when quality is costly: the persistence of the high-quality advantage. RA ND J ournal of Economics, 28, 372-84.

[12] M otta, M. (1993). Endogenous quality choice: price vs quantity competition. J ournal of Industrial E conomics, 41, 113-32.

[13] R onnen, U. (1991). M inimum quality standards, ${ }^{-}$xed costs, and competition. RAND J ournal of Economics, 22, 490-504.

[14] Rosenkranz, S. (1997). Quality improvements and the incentive to leapfrog. International J ournal of Industrial Organization, 15, 243-61. 
[15] Shaked, A. \& Sutton, J . (1982). R elaxing price competition through product di ßerentiation. Review of E conomic Studies, 49, 3-13.

[16] Shaked, A \& Sutton, J . (1983), Natural oligopolies. Econometrica, 51, 146983. 
n

\begin{tabular}{|c|c|c|}
\hline$q_{\mathrm{H}}^{\mathrm{n}}=0: 126655 \bar{\mu}^{4}=\mathrm{k}$ & $q_{H}^{H I}=0: 122232 \mu^{2}=k$ & $q_{H}^{L I}=0: 12667 \bar{\mu}^{2}=k$ \\
\hline$q_{L}^{n}=0: 024119 \bar{\mu}^{2}=k$ & $\mathrm{q}_{\mathrm{L}}^{\mathrm{HI}}=0: 023894 \bar{\mu}^{-2}=\mathrm{k}$ & $\mathrm{q}_{\mathrm{L}}^{\mathrm{LI}}=0: 024197 \bar{\mu}^{2}=\mathrm{k}$ \\
\hline $1 / 2 / 4=0: 012219 \bar{\mu}^{4}=k$ & $1 / 4{ }^{\prime \prime}=0: 012235 \bar{\mu}^{-4}=k$ & $1 /\left.4\right|^{\prime}=0: 0122069 \mu^{-4}=k$ \\
\hline $1 / \hat{t}=0: 000764 \bar{\mu}^{4}=k$ & $1 /\left.\right|^{\prime \prime}=0: 000757 \bar{\mu}^{4}=k$ & $1 /\left.\right|^{1}=0: 0007637 \bar{\mu}^{4}=k$ \\
\hline$S W^{n}=0: 034592 \mu^{-4}=k$ & $S W^{H I}=0: 034008 \mu^{4}=k$ & $S W^{\mathrm{LI}}=0: 034602 \bar{\mu}^{-4}=\mathrm{k}$ \\
\hline
\end{tabular}

Table 1 


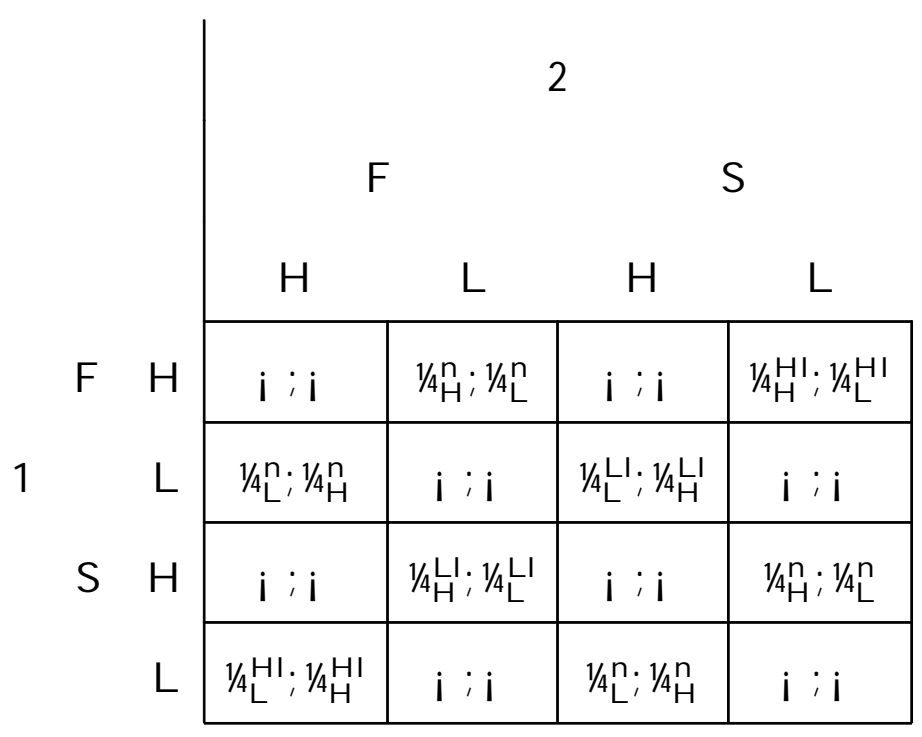

M atrix 1 


\begin{tabular}{c|c|c|c|}
\multicolumn{2}{|c}{2} \\
\multirow{2}{*}{1} & $\mathrm{~F}$ & $0: 064915 \bar{\mu}^{-4}=k ; 0: 064915 \bar{\mu}^{-4}=$ & $0: 064993 \bar{\mu}^{-4}=k ; 0: 064819 \bar{\mu}^{-4}=k$ \\
\cline { 3 - 5 } & & $0: 064819 \bar{\mu}^{-4}=k ; 0: 064993 \bar{\mu}^{-4}=k$ & $0: 064915 \bar{\mu}^{-4}=k ; 0: 064915 \bar{\mu}^{-4}=k$ \\
\cline { 3 - 4 } & & &
\end{tabular}

M atrix $1 b$ 


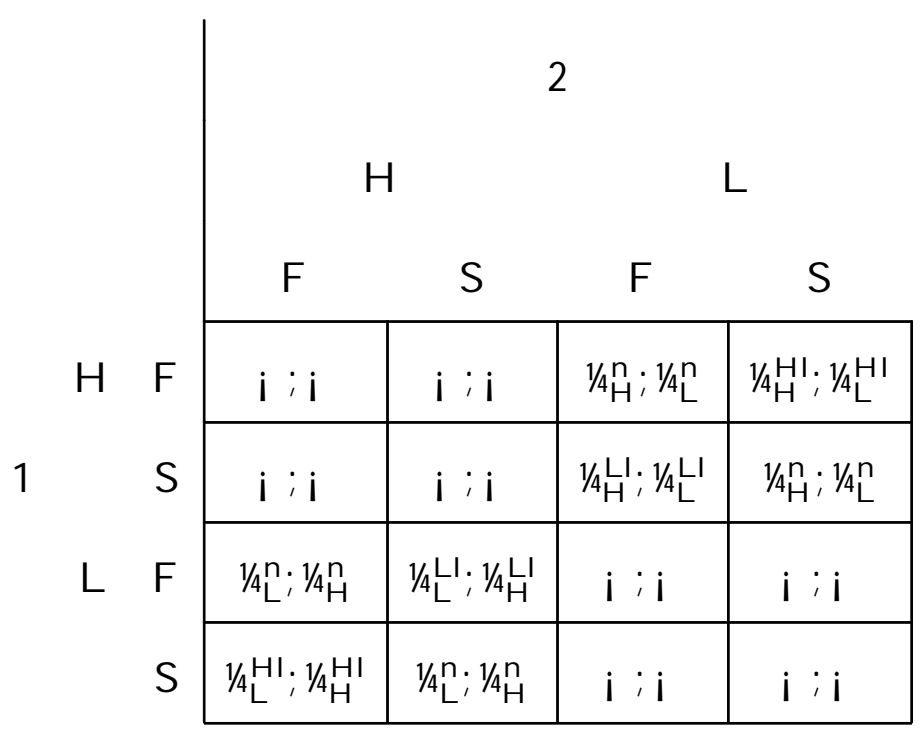

M atrix 2 


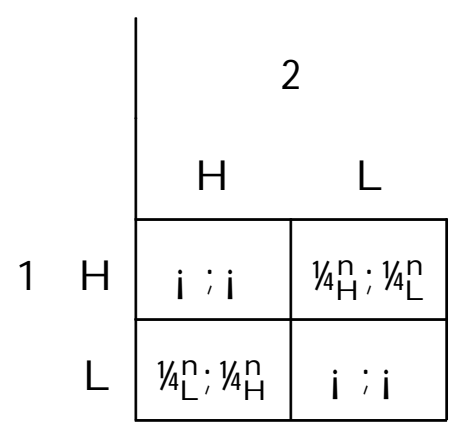

$M$ atrix 2b 